\title{
CYSTIC PITUITARY ADENOMATA
}

\author{
BY
}

\author{
J. E. PATERSON
}

(RECEIVED FEBRUARY 10, 1948)

\section{Review of the Literature}

References in the literature to cystic adenomata of the pituitary gland are by no means numerous. In a survey we have found surprisingly little discussion of this type of tumour, though it occurs with sufficient frequency to make its study of some practical importance from the point of view of both prognosis and treatment.

In Cushing's (1912) pioneer work on the surgery of the pituitary gland only one of his early operative series of twenty pituitary "strumas" is noted as cystic ; one other case, the famous giant, Turner (not operated on), was found at necropsy to have his pituitary adenoma almost entirely replaced by a large cyst. The small proportion of cysts in this early group of cases is curious, differing as it does so greatly from that found in other more recent series. Cushing was keenly alive to the very satisfactory decompression which should result from the evacuation of such cysts by the transphenoidal operation, and it seems unlikely that he failed to observe the escape of fluid when he incised the capsule of the gland from below.

When we pass to the modern era, with the operative approach predominantly transfrontal, we find a discussion of the cystic adenomata by Grant (1939), who emphasizes that these tumours have distinctive features from the standpoint of both prognosis and treatment, when compared with the solid growths. By far the most important and detailed report on the subject is Henderson's (1939) paper on the pituitary adenomata in Cushing's clinic, which deals with all the operative material from 1913 till 1932. He reported 338 cases of pituitary tumours, among which there were 49 with cyst formation.

It seems to be generally agreed that the cystic lesions show certain differences in behaviour when compared with the solid tumours. In particular it is stated that operations on the cystic cases result in a greater and more rapid improvement in the visual fields; that postoperative recurrence of visual field defects occurs more frequently; and that the cysts are more radio-resistant. Two varieties of cystic fluid are described; one clear, yellow in colour, the other dark reddish or brown and opaque. The proportion of cystic to solid tumours has been variously estimated at from 17 to 43 per cent., 17 per cent. is Henderson's figure for Cushing's series; Grant in a review of the ${ }^{0}$ literature found 25 per cent. to be cystic, but his own personal series contained 43 per cent., a figuree which, as we shall see later, is probably not com-8 parable with the others. The occurrence of an acute hæmorrhagic cyst has been described by Cushing (1926) and Sosman (1939). The latter reports a patient who after a course of $x$-ray treat ment for postoperative recurrence of a pituitary tumour went into coma in a few hours ; a hæmorrhagic cyst was found at operation and was evacuated with recovery.* The occurrence of this considerable proportion of cystic adenomata has practical importance if, as most writers believe, they are more radio-resistant than the solid growths, for this would provide an argument for the treatment of the pituitary adenomata by operation, rather than by radiation.

What should be our criterion of cyst formation when we expose the lesion at operation? Grant found 43 per cent. of his cases to be cystic, but he included tumours in which, on incision of the

* Since this paper was written we have encountered a case of acute hæmorrhage into a pituitary adenoma. A woman aged sixty-two had noticed for some months that the vision in her right eye was failing. Two evenings before admission she complained of headache and vomited several times that night. Next morning she was com pletely blind in both eyes. When examined next day the loss of vision was found to persist, with dilated fixed pupils and palsy of all the oculomotor nerves of both eyes except the left sixth. There was a typically ballooned sella in the radiographs. At operation on the day after admission a large chromophobe pituitary adenoma, with its dural capsule intact, was seen. It contained a large amount of blood-clot, some recent and some which, from its brown colour, its toughness, and its adherence to the tumour capsule, was thought to be of at least of some weeks' standing. After operation the patient remained in a stuporose state, from which she could be roused to carry out simple commands and to take nourishment, until her death three weeks later. There was no recovery of vision and the ocular palsies persisted. Towards the end the optic discs were becoming pale. 

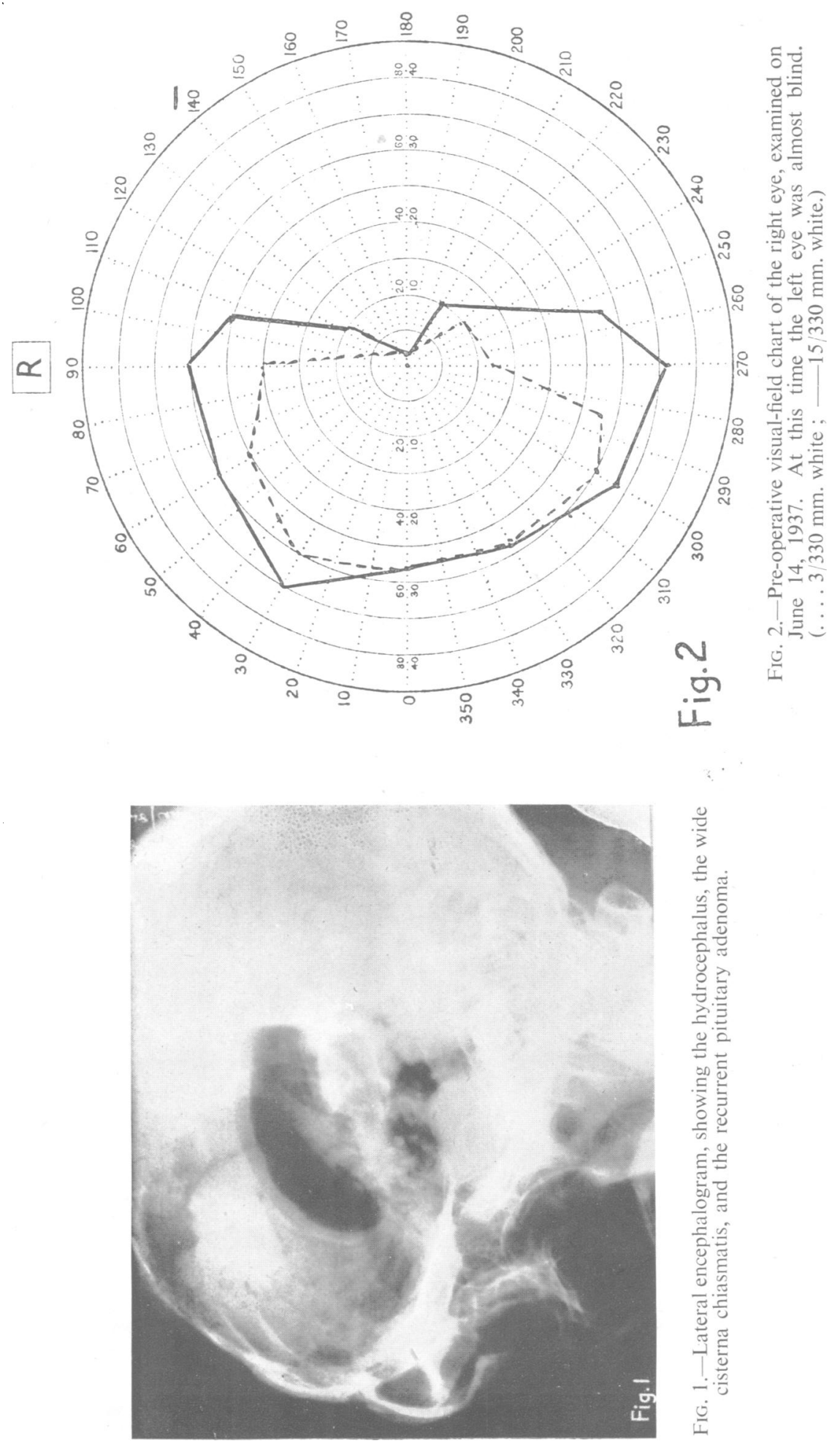


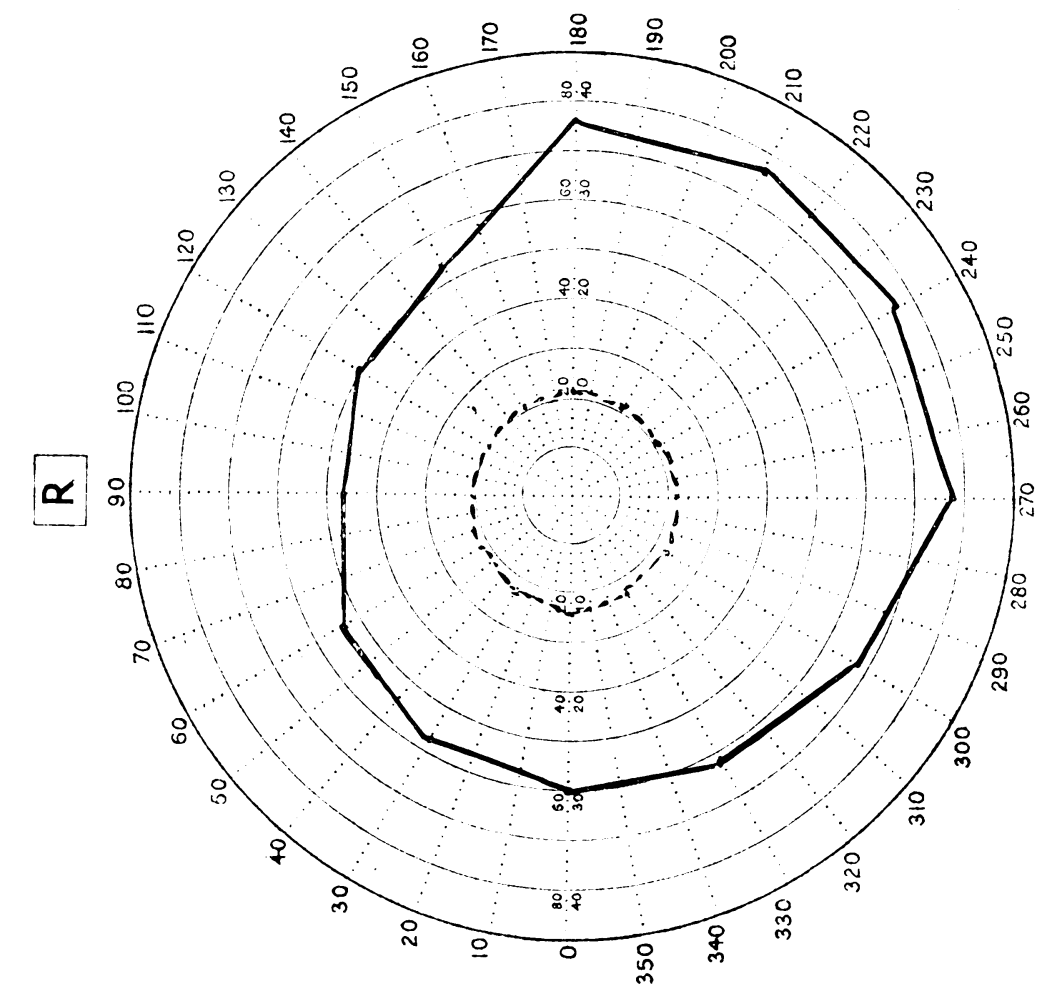

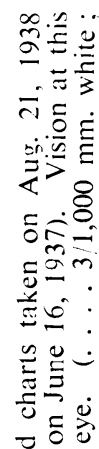

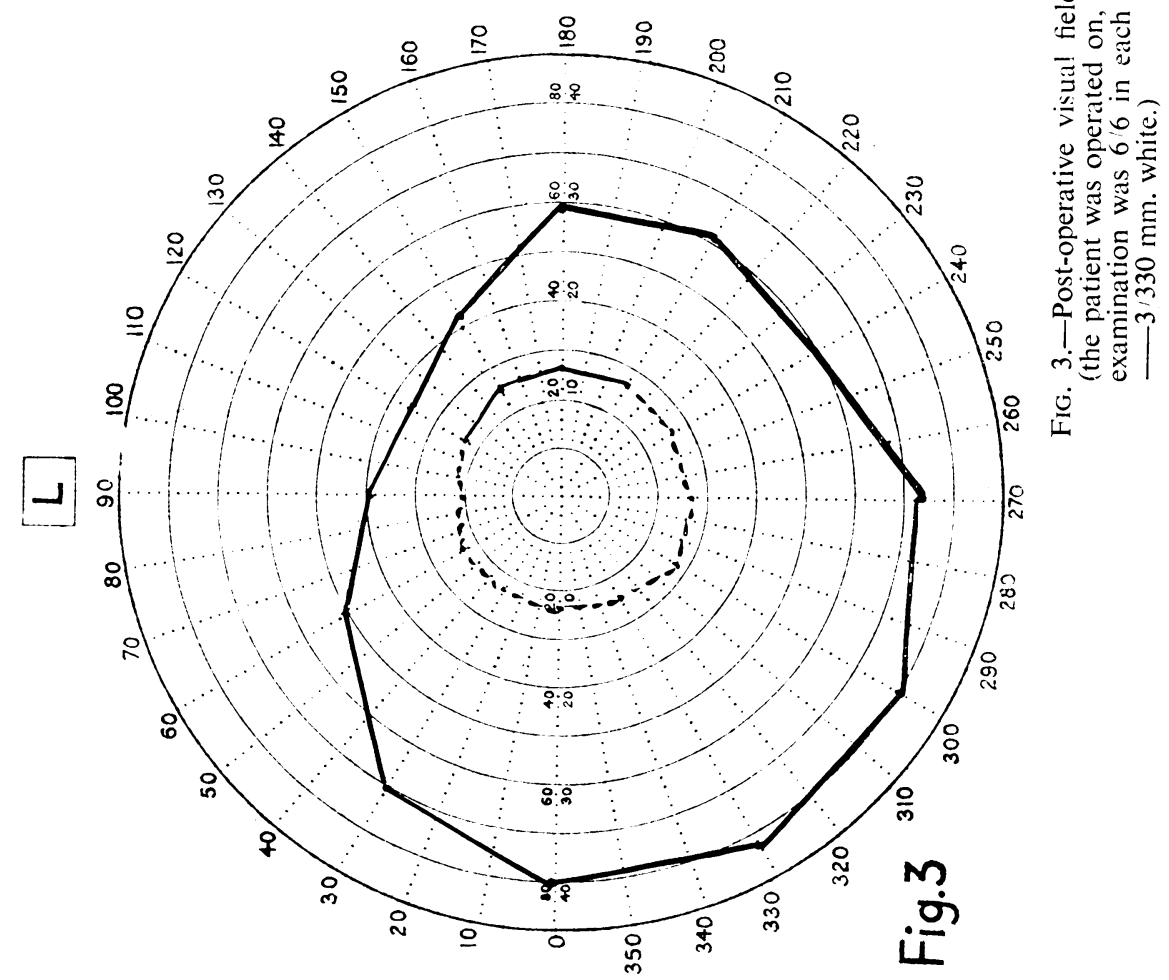




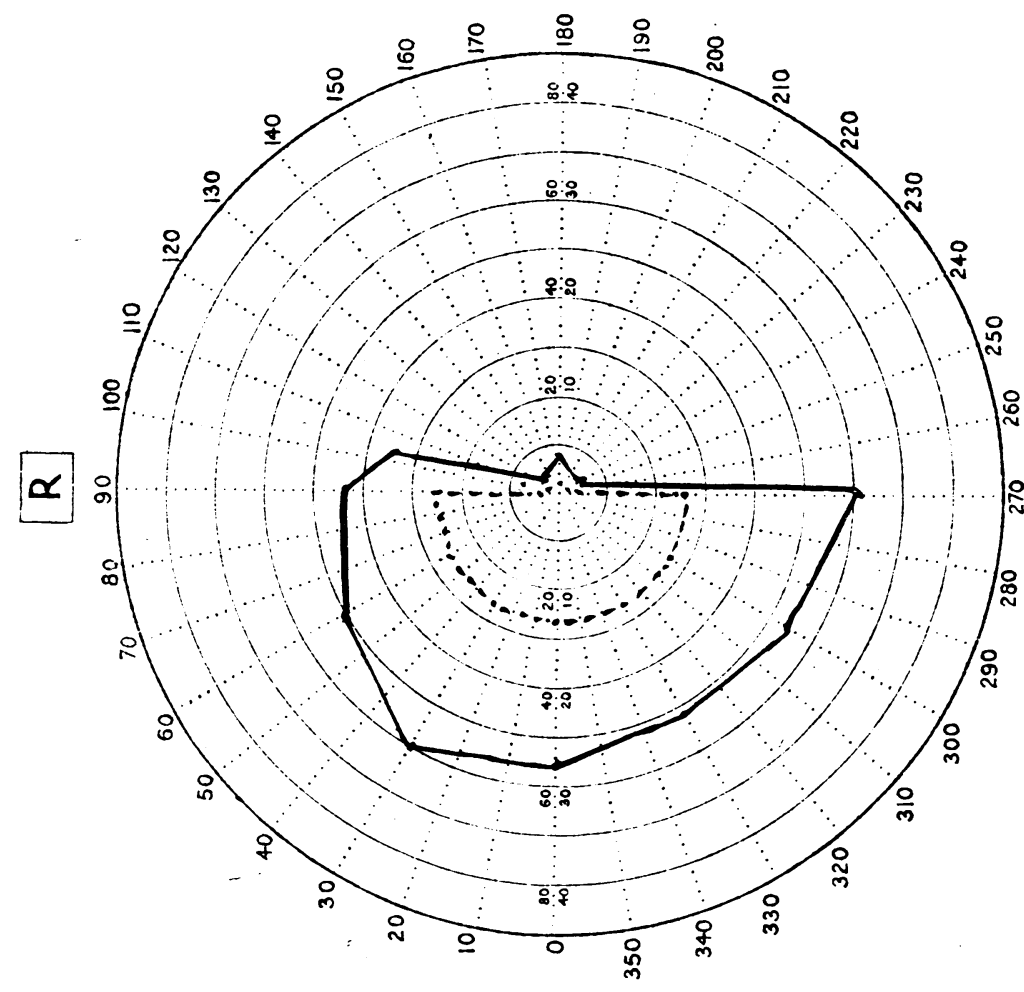

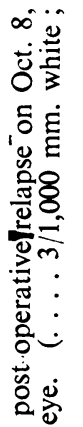

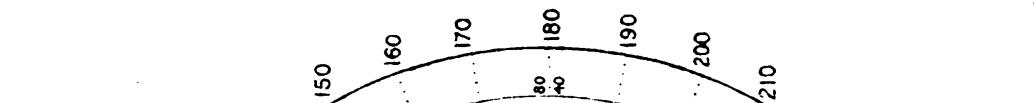

造过

起的畩

흥

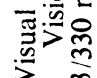

|बंगे।

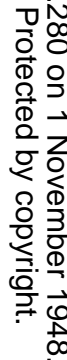

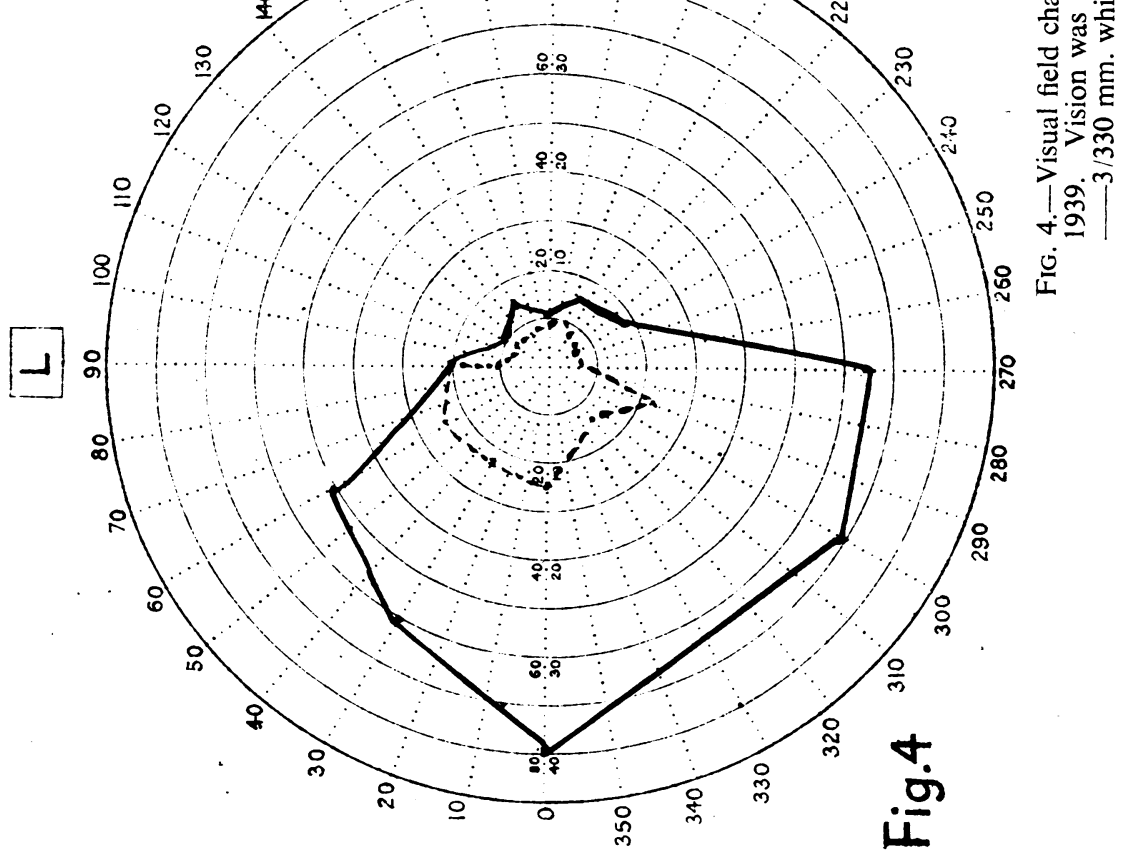

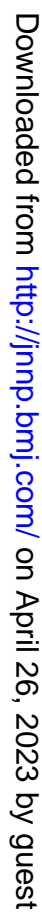




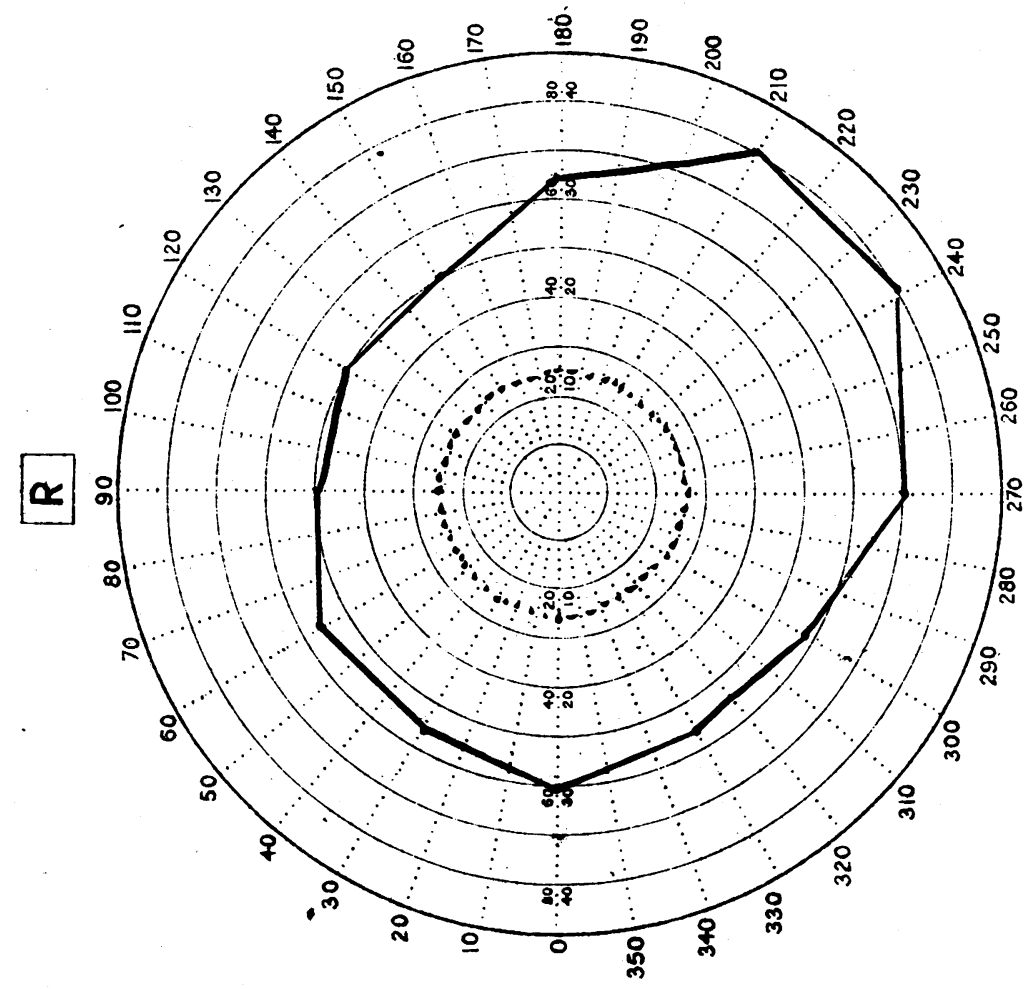

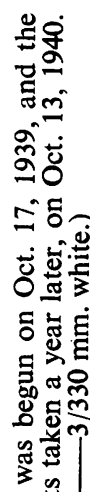

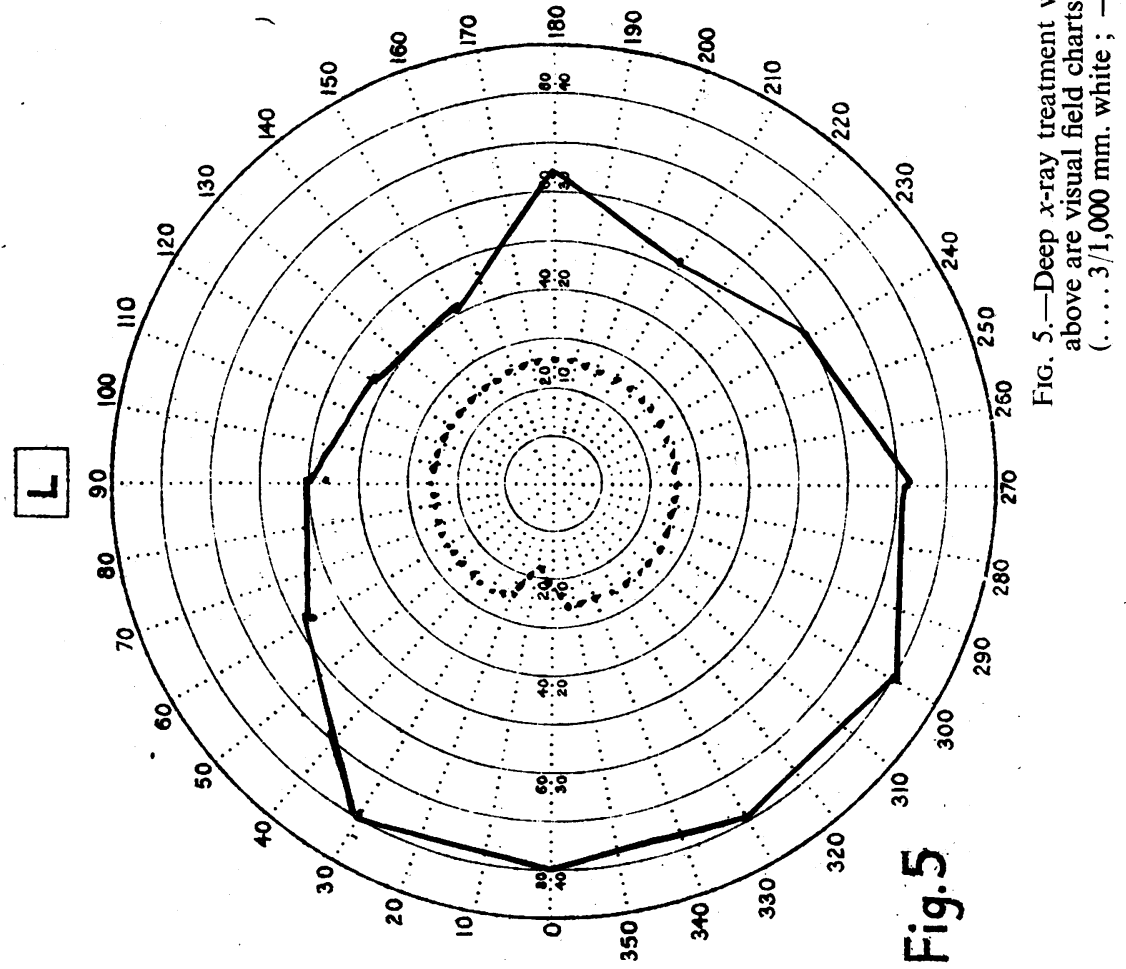


capsule, the contents flowed out " with prompt or slow collapse of the mass." This implies a very wide definition of the term cyst, for it must include a number of tumours with very soft contents, which protrude when the capsule is incised and which, following Jefferson (1940), we would rather class as diffluent. We reserve the term cystic for those tumours from which the operator can aspirate an appreciable amount of fluid through a spinal needle. Using this criterion a fair proportion at least of the contents must be fluid.

\section{The Present-Series}

If we define our cystic tumours in this fashion we find that, of forty-nine pituitary adenomata operated on, ten were cystic ( 20 per cent.). This proportion is not far removed from the 17 per cent. of Henderson's series.

Aspiration of the cystic fluid allows us to measure its quantity. Unfortunately we have no note of this in three of the cases, but in the remaining seven it was found to vary between 4 and $17 \mathrm{c.cm}$., with an average of about $10 \mathrm{c.cm}$.

Our experiences confirm that two distinct types of cyst content occur. In the first group the fluid is clear and yellow. The depth of the yellow coloration varies, much as it does in the cyst fluid of astrocytomas ; in one case it was so pale as to be almost colourless, while in others the colour was deep-golden orange. One example clotted spontaneously when allowed to stand in a test-tube, and it is possible that other specimens might have shown this property if they had been examined after standing. This spontaneous clotting is well known to occur in the fluid from cystic astrocytomas and hæmangioblastomas, and as we shall see later it may be produced by a similar mechanism. There were four cases with clear yellow fluid in the series. In the second group the fluid was dark and had sometimes the appearance of old blood, but in three tumours its characters were noted as typical of the contents of a Rathke's pouch cyst, brownish "motor-oil" fluid shimmering with cholesterol crystals. At one time we were under the impression that this type of fluid was pathognomonic of Rathke's cyst, and we feel it is important to appreciate that such brownish, cholesterol-containing cyst contents may occur in chromophobe pituitary adenomata. The operative and postoperative treatment of these two lesions is by no means identical, and the lesson to be drawn is that there should be a biopsy report in all such cases before operative closure.

With two exceptions, these cystic tumours were indistinguishable in their gross appearances from the solid ones, and were appreciated as cystic only when aspirated. One unusual finding was in the case of a girl with one eye blind and an advanced field defect in the other, whose clinical aspect was that of acromegaly with terminal hypopituitarism. The cyst wall was so densely calcified as to show up clearly in the radiographs; so dense was the calcification that bone rongeurs had to be employed to cut through the capsule after $13 \mathrm{c.cm}$. of thick, dark brown fluid had been aspirated. The other case was a chromophobe adenoma re-operated on for signs of recurrence two years after the primary operation. The wall of the large cyst was so thin as to appear bluish in colour; its contents were dark brown fluid.

There was one fatal case in the series. At operation a large cyst had yielded $16 \mathrm{c.cm}$. of brown fluid. Necropsy showed the lesion to extend backwards across the remains of the dorsum sella under the hypothalamus and to be distended with recent blood-clot; there had been a postoperative hæmorrhage into the cyst.

Biopsy Findings.-Biopsy specimens of nine of the ten cases showed histological appearances indistinguishable from those of the ordinary solid tumours ; eight were chromophobe adenomas, one acidophil. The only unusual picture was found in the fatal case just described, in which there were large thyroid-like vesicles, such as are seen in the pars intermedia of the normal gland. It is interesting that Cushing described such a tumour in his first monograph (1912). He pointed out the histological similarity to thyroid tissue, and suggested that some suprasellar growths may originate in the pars intermedia.

Cystic and Solid Tumours.-Is there any evidence in our series of cases that, as a review of the literature suggests, the adenomas with cystic contents are more liable to recur than the solid ones? Nine cystic and thirty-nine solid tumours survived operation. Of these it is known that four of the former and six of the latter had a recurrence of visual field defects. At first sight this seems to confirm the greater tendency to recurrence of the cystic adenomata, but the two series are not comparable in that, while the treatment of the solid growths was, from the first, operation followed by $x$ radiation, it was not until 1939, when the case reported below showed signs of recurrence, that radiation was used in the postoperative treatment of the cystic cases. We have thus no real evidence of the greater proneness to recurrence of the cystic growths.

Our experience is that recovery of vision and expansion of fields tend to be more rapid and more complete in the cystic cases. Failure of vision and visual-field defects in cases of tumour in the region 
of the chiasma are the result of changes in the visual pathways which may be either reversible or irreversible, and the extent of the postoperative recovery of vision must depend upon how complete is the relief of pressure on chiasma and optic nerves and upon the proportion of damage which is irreversible. It seems reasonable to assume that the amount of such damage must vary with the duration of the pressure on the chiasma and its blood vessels. In the slow-growing craniopharyngiomas the extent of the postoperative expansion of the visual fields is often very disappointing. The cystic adenomata, on the other hand, in which the operative relief of chiasmal pressure is complete and in which failure of vision is often of short duration, are among the most satisfactory of all chiasmal lesions ; they are above all others those in which, as Cushing pointed out long ago, the neurosurgeon shares with the ophthalmologist the supreme satisfaction of restoring vision. It seems reasonable to assume that this rapid and sometimes complete recovery of sight may be correlated with the rapid development of the cyst and a chiasmal pressure of short duration.

The Cyst Content.-Why are so many of these adenomata cystic, and why are the cyst contents sometimes a clear yellow fluid which may coagulate on standing and sometimes dark and opaque, like altered blood? The clear yellow fluid is similar to that found in the cystic astrocytomata and hæmangioblastomata, and it is suggested that a similar mechanism may be responsible for its production. The thin-walled tumour vessels may be unduly permeable to the blood plasma, allowing the passage not merely of albumen and globulin but, as in the case in which spontaneous clotting occurred, of fibrinogen as well. Where, on the other hand, the fluid is dark and opaque, we may imagine the vessel walls to have ruptured, with resulting hæmorrhage. Another factor may be a disturbance of the tumour circulation resulting from its enclosure in a rather unyielding fibrous and bony capsule; a state of affairs resembling that produced by a space-occupying intracranial lesion, in which a rise in venous pressure is an early feature. This high intracapsular pressure is often demonstrated by the extrusion, like tooth-paste from a tube; of the soft tumour contents when the capsule is incised, and a rise in venous pressure might be expected to encourage the escape of some or all of the elements of blood through the vessel walls.

The following case report is of interest as exemplifying the rapid and complete restoration of vision after operation, the tendency to relapse, and the danger of an overdose of $x$ radiation.

\section{Case Report}

A man aged 51 was seen first in 1937, when he presented the signs and symptoms of a chromophobe pituitary adenoma, with a temporal field defect of the right eye, and a blind left eye (Fig. 2.). At operation a cystic adenoma was found containing $17 \mathrm{c.cm}$. of dark fluid, obviously old blood. - He had no postoperative radiation at this time. After operation there was a rapid expansion of both fields of vision, which four months later were both full, with $6 / 6$ vision in each eye (Fig. 3). Two years after operation there was a relapse, and it is of interest that the visual fields now showed a right homonymous hemianopia of incongruous type, indicating pressure on the left optic tract (Fig. 4). Deep $x$-ray therapy was given, and there was once more a great expansion of the fields (Fig. 5). It seemed likely that there was a nodule of solid tumour, extending from the left lateral wall of the cyst; for it is difficult to imagine a cystic midline tumour sparing the chiasma so completely.

Another relapse occurred in 1943 ; operation was advised but was refused, and another course of $x$ radiation was given. There was very little improvement in the visual fields on this occasion, and thereafter the patient's general condition deteriorated. He was readmitted in 1945, when he was found to be bedridden and doubly incontinent, with signs of gross disturbance of hypo thalamic function, stupor, and mental confusion $-\infty$ Cisternal encephalography (Fig. 1) gave an interesting picture for the pituitary tumour was seen to be outline of by gas in a chiasmal cistern which, with the intere $z$ peduncular cistern, was grossly dilated, suggesting atrophy of the hypothalamus. Death occurred at home a few months later ; there was no necropsy.

It is difficult to avoid the conclusion that this terminat hypothalamic atrophy was the result of an overdose of $x$ rays ; the total dosage, calculated as delivered to the pituitary area, was $9,450 \mathrm{r}$.

\section{Summary}

Operative and postoperative experiences with ten cases of cyst formation in a group of forty-nine pituitary adenomata are reported. Cyst formation was found to occur in 20 per cent. of the series, and the cyst contents were found to be either a clear yellow, or a dark, brownish, opaque fluid. In three cysts in the latter group the fluid contained cholesterol crystals and was identical in naked-eye appearances with that found in cysts of Rathke's pouch. The importance of this finding in the differential diagnosis and treatment is indicated. Histologically the tumours, with one exception, were indistinguishable from other pituitary adenomata ; one was acidophil, the remainder chromophobe. The exceptional case showed large thyroidlike vesicles, suggesting an origin from the pars intermedia. Recovery of vision and expansion of fields were thought to be more rapid and complete in the cystic tumours than in the solid growths. The history is given of a patient in whom, after 
operation followed by great improvement in vision, two recurrences were treated by $x$ radiation, with subsequent signs and symptoms of fatal hypothalamic atrophy.

We wish to express our grateful thanks to Dr. Margaret Leslie, Pathologist, Killearn Hospital, who has examined all the biopsy material and has provided the photographic reproduction of the encephalogram.

\section{REFERENCES}

Cushing, H. W. (1912). "The Pituitary Body and its Disorders." J. B. Lippincott Co., Philadelphia. p. 304.

-(1926). "Studies in Introcranial Physiology and Surgery." Oxford University Press, London. p. 56. Grant, F. C. (1939). J. Amer. med. Ass., 113, 1279. Henderson, W. R. (1939). Brit. J. Surg., 26, 811. Jefferson, G. (1940). Proc. roy. Soc. Med., 33, 433. Sosman, M. C. (1939). J. Amer. med. Ass., 113, 1282.

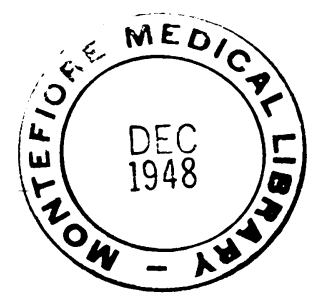

